\title{
Redefining amaurosis fugax
}

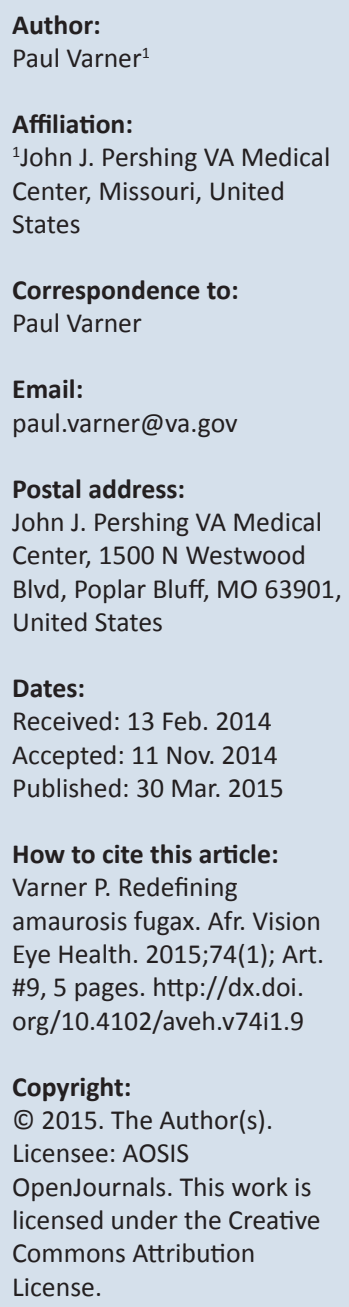
to read online.
Amaurosis fugax is a common term fraught with different interpretations. Disparities in understanding appear to be related to professional training. A new framework to facilitate interdisciplinary communication and clinical research is presented.

\section{Introduction}

Optometrists occasionally encounter patients who report marked, transient visual symptoms. Hypothetical examples include:

- A 26-year-old woman reports periodic 'white out' of the entire visual fields of both eyes that precludes visual resolution of any object during the events. These visual changes pass in approximately $15 \mathrm{~min}$, but are followed by an intense unilateral headache.

- A 63-year-old man reports three occurrences of vision that completely 'blacks out' his right eye during the preceding month. Vision in the left eye is unaffected during these incidents. Carotid auscultation is noteworthy for a bruit on the left side.

- An 81-year-old man presents with a recent history of episodic, short-lived, bilateral scintillating scotomata without associated headache or positional connection. During the acute phase, the patient can see gross hand movements, but is unable to discern large, individual optotypes.

In all three instances, the ophthalmic examination and neurological screenings are typically negative. What do these patients have in common?

The examples demonstrate just a few of the myriad presentations of transient vision loss, but do not necessarily present diagnostic dilemmas. The first example most likely represents classic migraine with visual aura, the second amaurosis fugax (AF) associated with carotid artery occlusive disease (CAOD), and the third is probably related to late-onset acephalgic migraine.

What is not apparent is that the visual symptoms reported in these illustrations may all represent variations of AF. Although AF is often considered to be a darkening or dimming of vision, such a restrictive definition depends more upon which sub-specialist is consulted than on interdisciplinary consensus. Consider the last example above: the cardiologist might deem such a presentation to be related to cardiac insufficiency from valvular disease. The ophthalmologist or optometrist might refer to this as an ophthalmic migraine. The neurologist might stop at a diagnosis of acephalgic migraine, whilst the vascular surgeon may perhaps not consider any of these diagnoses.

A medical literature review for AF reveals highly variable and inconsistent use of the terminology involved. Since diagnosis of these cases depends largely on clinical training, it is therefore intended that this report should serve as an entry point for an interdisciplinary resolution of this lexicon.

Clinically speaking, are the first and third examples veritable AF? More specifically, can AF (1) be bilateral or (2) present as scintillating visual aura - not as profound as dimmed sight or vision that has blacked out or turned gray? The answer to both questions appears to be 'yes', but is entirely dependent upon clinician training and the delineation of AF, so it seems evident that it is time to revisit some basic definitions.

\section{Background}

Etymologically, AF derives from the Greek terms amaurosis (amauroein) meaning 'to darken or obscure' and fugax (a Latinised version of the earlier Greek word pheugaleos) indicating 'to flee' or 'fleeting'. Historically, this phrase was used to denote many different kinds of vision problems, but it has acquired a more restrictive connotation in modern times. 
Fisher's seminal neurology paper, which appeared in the literature in 1951,1 associated 'fleeting blindness' with ipsilateral CAOD. Subsequent neurology reports concurred with this monocular designation, ${ }^{2}$ and it was re-emphasised in the recommendations of the Amaurosis Fugax Study Group. ${ }^{3}$ In the end, however, this was not an exclusive definition as the AF Study Group classified AF to be 'transient monocular visual loss attributed to ischaemia or vascular insufficiency' (italics added), but also acknowledged a form of AF that is bilateral. ${ }^{3}$ It should also be stressed that another important neurology study - the North American Symptomatic Carotid Endarterectomy Trial (NASCET) ${ }^{4}$ - concurred with this monocular designation. In view of the association of monocular AF with CAOD, vascular surgery has also tended to recognise AF as a unilateral phenomenon. ${ }^{5,6}$ The literature appears to be silent on the presence of patients with bilateral CAOD who might also have reported bilateral, yet asynchronous, AF. Perhaps this consideration may explain a lack of 'bilateral' case reports in the corpora of these disciplines.

Although AF has during the past 60 years become associated with a monocular sensation that is often directly related to $C A O D$, such restrictive use of the phrase is not ubiquitous. Case reports of 'bilateral amaurosis fugax' appear periodically, but seem more often to be reported in the cardiology literature. ${ }^{78,9,10}$ Amongst other cardiac sources of bilateral AF, mitral valve prolapse is a common cause of bilateral blurring of vision in older patients, ${ }^{11}$ although not necessarily in younger individuals. ${ }^{12}$ In older patients, the presumed relative ischaemia stems from decreased blood supply to the level of the Circle of Willis, but can also occur from other cardiac causes. ${ }^{13}$ When decreased cardiac output is not aetiologic, the anatomical basis for bilateral AF is more likely from vertebrobasilar insufficiency, as it is this circulation that is responsible for supplying the occipitus with its overlapping visual eye fields.

Ophthalmologists tend to refer to similar cases with bilateral visual symptoms as 'binocular transient vision loss'. ${ }^{14} \mathrm{AF}$ as briefly characterised by Duke-Elder in the classic ophthalmic reference involves 'temporary obscurations of vision', ${ }^{15}$ but did not restrict the occurrence by laterality or extent of vision loss. Ophthalmology has not further delineated AF by laterality or level of vision loss ${ }^{16}$ as have other sub-specialties. It has been further suggested that this term is inaccurate in describing instances of complete visual loss. ${ }^{17}$

Thus it appears that current clinical interpretation of AF depends on specialty training. In general, neurologists and vascular surgeons associate AF with transient monocular vision loss (completely dark vision) related to ipsilateral CAOD; cardiologists tend to consider $\mathrm{AF}$ as transient binocular blurring of vision (not necessarily dark vision) that can be related to cardiovascular insufficiency; and ophthalmologists use AF as a catch-all phrase for many forms of transitory visual change in one or both eyes (across the entire spectrum of light and dark visual compromise). These generalisations are characterised by the patient's subjective degree of reported visual loss during the acute phase of visual symptoms. Owing to the transient nature of AF, nearly all patients with this ocular complaint are evaluated after the visual phenomena have resolved. Therefore it is extremely difficult to determine the extent of vision loss during some AF episodes for the majority of patients. Perhaps this paucity of data - in addition to perceived disparate clinical outcomes has contributed to a seemingly intuitive separation of patients with dark vision incidents from those with light vision experiences.

\section{Discussion}

Visual acuities have occasionally been reported during dark vision events. These indicate either light perception $(\mathrm{LP})^{18}$ or no light perception (NLP) $)^{19,20,21}$ during the acute phase of AF. Only Bougousslavsky is from a non-ophthalmology source (cardiology), but these findings reinforce the suggestion that most clinicians associate AF with profound vision loss prior to return to baseline visual status. Vision levels during scintillations appear to be unreported; the literature review for this article revealed no reports addressing AF for blurred vision less than LP or NLP vision.

Rather than considering $\mathrm{AF}$ as a pathognomonic occurrence, perhaps the time has come to reconsider it as a symptom of a number of underlying conditions. Even for 'idiopathic' cases, neither light-vision nor dark-vision AF is a normal, expected finding. It may be most instructive to consider AF from the broader standpoint of transience, especially considering the wide-ranging continuum of clinical presentations with variable laterality (monocular vs. binocular), frequency of association (CAOD), degree of vision loss (dim or excessively blurred vision), and the length of vision compromise (a few seconds to a few hours). AF need not be restricted to refer solely to complete vision loss (NLP or LP) - rather is it a transient obscuration of vision (in the broadest interpretation of the Greek terms) with return to baseline function.

There is a great subjective difference between patients who experience bilateral blurring of vision (can still see objects) and those who experience total vision loss (unable to see anything). It seems likely that subjects with more profound decreases in vision (dark vision) would be more apt to seek medical care than those with excessive blurring of vision (the equivalent of being unable to accurately report even the largest visual acuity chart optotypes), perhaps also explaining why vision levels during light-vision events are underreported. This consideration is likewise possibly related to objective morbid outcomes associated with darkvision occurrences as compared with relative benignity of light-vision phenomena.

Whilst most references associate AF with blackening or dimming of vision, this may be too restrictive if one considers the underlying source for the ephemeral visual changes: namely, ischaemia. It seems apparent that either type of visual symptomatology (dimming or excessive blur) is 
related to a hypoxic antecedent - either of which can produce 'dimmed' or 'blurred' vision in either or both eyes.

$\mathrm{AF}$ is most accurately reflective of an ophthalmic transient ischaemic attack (TIA). The American Heart Association and American Stroke Association (AHA and ASA) currently define TIA as 'a transient episode of neurological dysfunction caused by focal brain, spinal cord, or retinal ischaemia, without acute infarction'. ${ }^{22}$ Clearly, short-lived visual disturbances related to retinal or cortical hypoxia fall into this category and need not be restricted by laterality of symptomatology. Nor does this definition limit retinal ischaemia to internal carotid antecedents. It therefore seems prudent to consider AF as a TIA, regardless of monocular or binocular presentation, or the type of visual symptom. This stance includes the visual spectrum from an aura involving excessively blurred vision (discussed below) through no vision (dim, dark, gray, blackened vision, transient 'blindness') during the acute phase - with return to baseline function. Variability of subjective reports has been noted during migraine and TIA events, ${ }^{23,24}$ and it may be conjectured that visual compromise ultimately depends on the extent of relative oxygen deprivation. Duration of altered sensation - from a few minutes to a few hours - also seems likely to be related to the time interval of cortical ischaemia.

Within this context, the degree of visual symptoms may be considered. AF does not include minimal visual blurring but, rather, significant visual blur (no better than 20 out of 400) in the presence of light-field or positive phenomena. Positive experiences can include migraine auras (scintillations, fortifications, twinkling lights or stars, whiteout, etc.), and represent any addition to the visual perceptual fields. For these presentations, perhaps phos AF would be a more accurate descriptor - phos being the Greek transliteration for 'light,' indicating positive or light portents.

Conversely, dim, dark or gray vision would include darkfield or negative visual sensations - a lack of visual perceptual field stimulation. Skotos is the Greek transliteration of 'dark', and indicates the lack of visual input. Essentially, patients with skotos AF perceive nothing in their visual fields and are describing NLP (or rarely LP) vision. These Greek derivatives for light or dark transient obscurations of vision are analogous to ophthalmic use of descriptors for photopic and scotopic vision. This usage is similarly consistent with neurological designations for positive and negative visual auras associated with migraine events. ${ }^{25}$

\section{Differential diagnosis}

Figure 1 depicts a differential diagnosis for AF that encompasses both light- and dark-vision symptomatology. Paradoxical presentations may defy characterisation; however, some generalisations can be used to assist providers in various clinical settings. After determination of phos versus skotos AF, laterality of symptoms is established. For phos $\mathrm{AF}$, the presence of cephalgia helps to determine the diagnosis.

The International Headache Society (IHS) has strictly defined retinal migraine (a.k.a. ophthalmic migraine) as fullyreversible monocular visual disturbances associated with

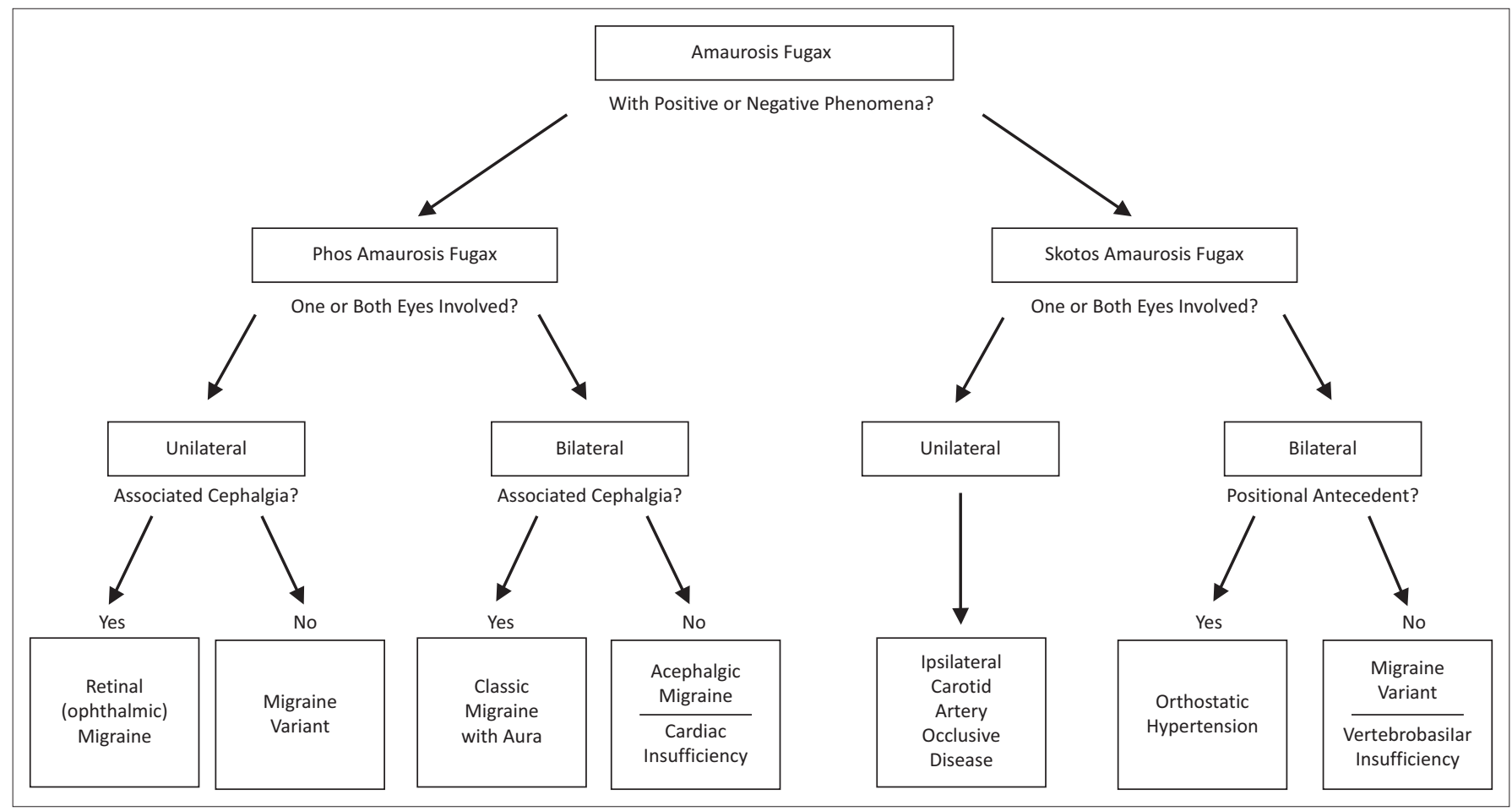

Source: Created by Ms Antonia Varner

FIGURE 1: Differential diagnosis of phos and skotos amaurosis fugax. 
typical migraine headache with normal neuro-ophthalmic examination between attacks, with 'visual disturbances' including 'blindness' ${ }^{26}$ Whilst the source for retinal migraine is considered to be vasospastic, cases that strictly meet IHS criteria are exceedingly rare, ${ }^{27}$ and it has been suggested that the term 'retinal migraine' can be misleading. ${ }^{28}$ Because of stringent IHS criteria for retinal migraine, cases of monocular phos AF without concurrent cephalgia may be considered migraine variants.

Bilateral phos AF associated with cephalgia is considered a harbinger of classic migraine with aura, and is illustrated by the first example in this report. However, when bilateral migraine auras occur without headache, the condition is termed acephalgic migraine. Late-onset cases of acephalgic migraine are not unusual, and are not heralds of cerebrovascular accident (CVA) ${ }^{29}$ (the third example above). Rarely, cardiac insufficiency can be associated with these bilateral phenomena, so cardiovascular consultation may be considered in patients with late-onset bilateral auras without hemicranias.

The differential diagnosis of skotos AF also relies on determination of laterality. Unilateral cases are highly suggestive of ipsilateral CAOD and merit carotid studies, although only $20 \%$ - $50 \%$ of patients may have positive findings ${ }^{30,31}$ (this is exemplified by the second example). Bilateral symptoms require further query regarding body positioning. When subjective reports can be linked to postural changes, then orthostatic hypotension is to be expected. Without positional antecedent, then migraine variant and vertebrobasilar insufficiency should be considered, although the medical literature is frustratingly silent on the latter of these two diagnostic possibilities.

\section{Unanswered questions}

It should be emphasised that there is an extremely wide variety of temporary visual complaints reported by patients. ${ }^{32,33}$ Variability may be related to physiology, but this is poorly understood at present. The physiological difference between visual complaints reported by patients with phos AF (fleeting light vision) and skotos AF (fleeting dark vision) may be because of the degree of relative ischaemia of involved ocular or cortical tissue. This area remains unstudied.

Furthermore, some cases of AF remain 'idiopathic', even in the presence of comprehensive medical work-up.,34 These enigmatic presentations defy identification of underlying cause or clear association and represent the boundary of current medical knowledge. No source for skotos AF will be discovered in approximately 1 in 10 cases of AF amongst patients under the age of $50,{ }^{3}$ whilst that for older patients is unclear. Aetiologies for phos AF events are unreported.

No individual professional societies have published formal guidelines regarding transient visual symptoms across the entire spectrum of ophthalmic compromise. The Amaurosis
Fugax Study Group recommended ophthalmic examination, basic laboratory studies and carotid duplex ultrasonography for skotos AF, and a 13-profession interdisciplinary consensus statement advises cartoid endarterectomy for skotos AF patients when AF is associated with $\geq 70 \%$ stenosis by noninvasive imaging ${ }^{35}$ for patients with these same complaints.

\section{Conclusion}

In fine, $\mathrm{AF}$ is not pathognomonic or a final diagnosis in and of itself. AF is best understood as an indicator of underlying disease that represents a spectrum of fleeting visual obscurations ranging from excessive blur to complete vision loss of variable duration, and is characterised by a return to baseline function between episodes. AF phenomena can be positive or light or negative or dark, unilateral or bilateral, and should be considered as an ophthalmic TIA, consistent with the AHA and ASA definition. The underlying theme of AF is simply transience - not laterality or the specific visual symptoms.

As an ocular TIA, AF requires further delineation prior to differential diagnosis of the underlying aetiology. Understanding these characteristics will assist in the management of these patients who present in the clinics of several different specialists. Redefining AF by separating phos AF from skotos AF might augment clinical communication and help to improve differential diagnoses. An initial scheme for the work-up of these patients is provided, and it is hoped that this will be modified as multidisciplinary, clinical understanding of AF improves. This report is intended to help to bridge interdisciplinary gaps in terminology related to the spectrum of AF as a TIA.

\section{Acknowledgements}

I thank Ms Antonia Varner for the creation of Figure 1.

\section{Competing interests}

The author declares that he has no financial or personal relationships which may have inappropriately influenced him in writing this article.

\section{References}

1. Fisher CM. Occlusion of the internal carotid artery. Arch Neurol Psych. 1951;65:346377. http://dx.doi.org/10.1001/archneurpsyc.1951.02320030083009

2. Wilson LA, Russell RWR. Amaurosis fugax and carotid artery disease: Indications for angiography. Br Med J. 1977;2:435-437. http://dx.doi.org/10.1136/bmj.2.6084.435

3. The Amaurosis Fugax Study Group. Current management of amaurosis fugax. Stroke. 1990;21:201-208. http://dx.doi.org/10.1161/01.STR.21.2.201

4. Benavente O, Eliasziw M, Streifler JY, Fox AJ, Barnett HJM, Meldrum, for the North American Symptomatic Carotid Endarterectomy Trial Collaborators. Prognosis after transient monocular blindness associated with carotid-artery stenosis. $\mathrm{N}$ Engl J Med. 2001;345:1084-1090. http://dx.doi.org/10.1056/NEJMoa002994

5. Torem S, Rossman ME, Schneider PA, Otis SM, Dilley RB, Bernstein EF. The natural history of amaurosis fugax with minor degrees of internal carotid artery stenosis. Ann Vasc Surg. 1990;4:46-51. http://dx.doi.org/10.1007/BF02042689

6. Rosenthal D, Hungerpillar JC, Crispin ME, Clark MD, Lamis PA, Pallos LL. Amaurosis fugax: Is it innocuous? Ann Vasc Surg. 1992;6:281-288. http://dx.doi.org/10.1007/ BF02000275

7. Schievink WI, Bjornsson J, Piepgras DG. Coexistence of fibromuscular dysplasia and cystic medial necrosis in a patient with Marfan's syndrome and bilateral carotid artery dissections. Stroke. 1994;25:2492-2496. http://dx.doi.org/10.1161/01. STR.25.12.2492 
8. Araya-Munoz CE, Lopezcarasa-Hernandez G, Romero-Castro RM, Amigo C, Jimenez-Sierra JM. Antiphospholipid syndrome diagnosed by ocular vascular occlusion as the first clinical manifestation: Report of three cases. Invest occlusion as the first clinical manifestation:
Ophthalmol Vis Sci. 2001;43: E-abstract 499.

9. Benchimol-Barbosa PR, Vilele FD, Alves de Melo FB, Cordiero da Rocha AS. A 53-year-old male with bilateral carotid occlusion and a functionally preserved cerebral circulation due to compensatory bilateral veterbral artery flow and reversed bilateral flow through the superior thyroid arteries. Rev Esp Cardiol. 2008;61:1355-1365.

10. Pleşa C, Grigorescu A, Tantu M, Cristina S. Diaconu M. Amaurosis fugax an alarm signal for cerebral vascular accident. Acta Medica Transilvanica. 2010;2:221-222.

11. Walsh PN, Kansu TA, Corbett JJ, Savino PJ, Goldburgh WP, Schatz NJ. Platelets, thromboembolism and mitral valve prolapse. Circulation. 1981;63:552-559. http://dx.doi.org/10.1161/01.CIR.63.3.552

12. Tippin J, Corbett JJ, Kerber RE, Schroeder E, Thompson HS. Amaurosis fugax and ocular infarction in adolescents and young adults. Ann Neurol. 1989;26:69-77. http://dx.doi.org/10.1002/ana.410260111

13. Paelinck BP, De Raedt $H$, Conraads V. Blurred vision, left bundle-branch block and cardiac failure. Acta Cardiol. 2001;56:39-40. http://dx.doi.org/10.2143/ AC.56.1.2005592

14. Burde RM, Trobe JD, Savino PJ. Transient visual loss. In: Burde RM, Trobe JD, Savino PJ, editors. Clinical decisions in neuro-ophthalmology. 3rd ed. New York: Elsevier Health Sciences, 2002; p. 94-113.

15. Duke-Elder S. Disturbances of the circulation. In: Duke-Elder S, editor. System of Ophthalmology. Vol X: Diseases of the retina. St Louis: The CV Mosby Company, 1967; p. 43-198.

16. Burde RM. Amaurosis fugax: An overview. J Clin Neuroophthalmol. 1989;9: 185-189.

17. Wilhelm H. Transient visual loss. In: Clinical neuro-ophthalmology. Berlin: Springer 2007; p. 195-201. http://dx.doi.org/10.1007/978-3-540-32708-0_14

18. Mimura T, Funastsu H, Kitano S, et al. Diabetic retinopathy with repeated amaurosis fugax caused by orthostatic hypotension. Am J Ophthalmol. 2003;136:930-931. http://dx.doi.org/10.1016/S0002-9394(03)00894-8

19. Bogousslavsky J, Vinuela F, Barnett HJM, Drake CG. Amaurosis fugax as the presenting manifestation of dural arteriovenous malformation. Stroke. 1985;16: presenting manifestation of dural arteriovenous mal
891-893. http://dx.doi.org/10.1161/01.STR.16.5.891

20. Narita AS, Edler JE. Ocular migraine in an eight-year-old girl. Aust N Z J Ophthalmol. 1994;22:275-277. http://dx.doi.org/10.1111/j.1442-9071.1994.tb00797.x

21. Doyle E, Vote BJ, Casswell AG. Retinal migraine: Caught in the act. Br J Ophthalmol. 2004;88:301-302. http://dx.doi.org/10.1136/bjo.2003.021808

22. Easton JD, Saver JL, Albers GW, et al. Definition and evaluation of transient ischaemic attack: A scientific statement for healthcare professionals from the American Heart Association/American Stroke Association Stroke Council; Council on Cardiovascular Surgery and Anesthesia; Council on Cardiovascular Radiology and Intervention; Council on Cardiovascular Nursing; and the Interdisciplinary Council on Peripheral Vascular Disease. Stroke. 2009;40:2276-2293. http://dx.doi. org/10.1161/STROKEAHA.108.192218
23. Russell MB, Olesen J. A nosographic analysis of the migraine aura in a general population. Brain. 1996;119:355-361. http://dx.doi.org/10.1093/brain/119.2.355

24. Amort M, Fluri F, Schäfer J, et al. Transient ischaemic attack versus transient ischaemic attack mimics: Frequency, clinical characteristics and outcome. Cerebrovasc Dis. 2011;32:57-64. http://dx.doi.org/10.1159/000327034

25. Marks DDR, Rapoport AM. Diagnosis of migraine. Semin Neurol. 1997;17:303306. http://dx.doi.org/10.1055/s-2008-1040942

26. Headache Classification Committee of the International Headache Society (IHS). The international classification of headache disorders. 3rd ed. Cephalgia. 2013;33:629-808.

27. Hill DL, Daroff RB, Ducros A, Newman NJ, Biousse V. Most cases labeled as 'retinal migraine' are not migraine. J Neuro-Ophthalmol. 2007;27:3-8. http://dx.doi. org/10.1097/WNO.0b013e3180335222

28. Winterkorn JMS. 'Retinal migraine' is an oxymoron. J Neuro-Ophthalmol. 2007;27:1-2. http://dx.doi.org/10.1097/WNO.0b013e3180334dd1

29. Wijman CAC, Wolf PA, Kase CS, Kelly-Hayes M, Beiser AS. Migrainous visual accompaniments are not rare in late life: The Framingham study. Stroke. 1998;29:1539-1543. http://dx.doi.org/10.1161/01.STR.29.8.1539

30. Donders RC, Dutch TMBStudy Group. Clinical features of transient monocular blindness and the likelihood of atherosclerotic lesions of the internal carotid artery. I Neuro Neurosurg Psychiatry. 2001;71:247-249. http://dx.doi.org/10.1136/jnnp.71.2.247

31. McCullough HK, Reinert CG, Hynan LS, et al. Ocular findings as predictors of carotid artery occlusive disease: Is carotid imaging justified? J Vasc Surg. 2004:40:279286. http://dx.doi.org/10.1016/j.jvs.2004.05.004

32. Gaul JJ, Marks SJ, Weinberger J. Visual disturbance and carotid artery disease: 500 symptomatic patients studied by non-invasive carotid artery testing including b-mode ultrasonography. Stroke. 1986;17:393-398. http://dx.doi. org/10.1161/01.STR.17.3.393

33. Vincent $M B$, Hadjikhani N. Migraine aura and related phenomena: Beyond scotomata and scintillations. Cephalgia. 2007;27:1368-1377. http://dx.doi. org/10.1111/j.1468-2982.2007.01388.x

34. Newman NJ. Cerebrovascular disease. In: Walsh and Hoyt's Clinical Neuroophthalmology. 5th ed. Baltimore: Lippincott, Williams \& Wilkins, 1998; p. 3323-3656.

35. Brott TG, Halperin JL, Abbara S, et al. 2011 ASA/ACCF/AHA/AANN/AANS/ACR/ ASNR/CNS/SAIP/SCAI/SIR/SNIS/SVM/SVS guideline on the management of patients with extracranial carotid and vertebral artery disease: A report of the American College of Cardiology Foundation/American Heart Association Task Force on Practice Guidelines, and the American Stroke Association, American Association of Neuroscience Nurses, American Association of Neurological Surgeons, American College of Radiology, American Society of Neuroradiology, Congress of Neurological Surgeons, Society of Atherosclerosis Imaging and Prevention, Society for Cardiovascular Angiography and Interventions, Society of Interventional Radiology, Society of Neurolnterventional Surgery, Society for Vascular Medicine, and Society for Vascular Surgery. Circulation. 2011;124:e543130. http://dx.doi.org/10.1161/CIR.0b013e31820d8d78 